\title{
Redução de incertezas em análise de vulnerabilidade às mudanças climáticas para Dicksonia sellowiana
}

\author{
Marcelo Brilhante de Medeiros ${ }^{1}$, Mahalia Sojo Cardoso², Rafael Walter Albuquerque ${ }^{2}$, Sérgio Eustáquio Noronha1 \\ ${ }^{1}$ Embrapa Recursos Genéticos e Biotecnologia, Parque Estação Biológica - PqEB - Av. W5 Norte, CP 02372, CEP 70770-917, Brasília, DF, Brasil \\ 2Universidade de Brasília, Departamento de Engenharia Florestal, Asa Norte, CEP 70910-900, Brasília, DF, Brasil
}

\author{
*Autor correspondente: \\ marcelo.brilhante@embrapa.br \\ Termos para indexação: \\ Mata Atlântica \\ Xaxim \\ Aquecimento global \\ Nicho ecológico \\ Index terms: \\ Atlantic Forest \\ Xaxim \\ Global warming \\ Ecological niche
}

Histórico do artigo:

Recebido em 28/02/2012

Aprovado em 01/07/2013

Publicado em 30/09/2013
Resumo - Este estudo teve por objetivo estabelecer predições do impacto das mudanças climáticas em cenários futuros sobre Dicksonia sellowiana (Pres.) Hook, por meio de modelos de distribuição de espécies e uso de técnicas que diminuam o grau de incerteza nesse tipo de modelagem. Para a geração dos modelos foram utilizados cinco algoritmos disponíveis no programa Openmodeller. Amodelagem incluiu o modelo climático HadCM3, com projeções para o ano 2050, em dois cenários de emissão de CO2: pessimista (A2) e otimista (B2). Todos os algoritmos apresentaram eficiência nas projeções para o clima atual, o que foi validado pelos valores da área sobre a curva ROC e pelo índice de Kappa. Considerando os remanescentes de habitats disponíveis para a espécie, os resultados apresentaram diferenças significativas entre as projeções de área com o clima atual e em relação às áreas em cenários climáticos futuros. Esses modelos indicaram reduções de área potencial superiores a $50 \%$ para a espécie. Mesmo considerando os valores bons e excelentes de acurácia com os dados de clima atual, a variabilidade das projeções de distribuição para o clima no futuro indica a necessidade do uso de técnicas de consenso para a redução das incertezas associadas a este tipo de modelagem. for Dicksonia sellowiana (Pres.) Hook

\begin{abstract}
This work had the objective of investigate the impacts of climate changes with different climate settings on the spatial distribution of Dicksonia sellowiana (Pres.) Hook. The survey was carried out by using spatial distribution modeling and methods in order to reduce the uncertainty of these predictions. The software Openmodeller was used to run the modeling with five spatial distribution algorithms. The modeling included the climate model HadCM3 of green house gases emission for the year 2050 for two CO2 emission scenarios: optimistic (B2) and pessimistic (A2). The area under the curve (AUC statistics) and the Kappa statistics resulted high values for all algorithms tested meaning high values of accuracy. Regarding only the species habitats available all algorithms have shown efficient distribution models and the projections were significantly different between the predictions for the future climate and for the current climate. Both models have shown reduction of $50 \%$ of the species potential area . Even with the high values of accuracy the variability of predictions for the future spatial distribution models confirm the need of consensus methods to reduce the uncertainty associated with this type of modeling.
\end{abstract}




\section{Introdução}

As respostas das espécies às mudanças climáticas podem incluir extinções, adaptações ou mudanças nas amplitudes de distribuição geográfica (Diniz-Filho \& Bini, 2008). A conservação de nicho, ou a tendência das espécies em reter características do nicho fundamental (sensu Hutchinson, 1957), é um dos fatores que podem tornar as mudanças climáticas potencialmente impactantes para a biota mundial (Wiens \& Graham, 2005). Alguns estudos demonstraram que muitas espécies respondem às mudanças climáticas com conservação de nichos, ao contrário de mudanças evolutivas rápidas para maior tolerância climática (Parmesan \& Yohe, 2003).

Considerando a premissa de que o clima desempenha um papel fundamental no limite de distribuição das espécies (Pearson \& Dawson, 2003), o uso da modelagem tem permitido a simulação de impactos de mudanças climáticas sobre a biota (Guisan \& Thuiller, 2005; Araújo et al., 2005a, 2006; Algar et al., 2009), com evidências de mudanças substanciais na distribuição de espécies (Siqueira \& Peterson 2003; Colombo \& Joly 2010) e estimativas de impacto negativo relevante para o processo de extinção de espécies (Peterson et al., 2002; Thomas et al., 2004).

Porém, algumas análises têm demonstrado que ainda há muitas incertezas que devem ser consideradas no processo de modelagem, e que podem ser reduzidas com a adoção de critérios mais específicos no uso de diferentes modelos, na seleção dos dados geográficos e climáticos e nos cenários de emissão de gases de efeito estufa (Thuiller, 2004; Guisan \& Thuiller, 2005; Pearson et al., 2006; Beaumont et al., 2007). Desconsiderar o potencial das incertezas pode se tornar um problema porque, entre outras razões, o planejamento para conservação biológica precisa se basear em predições mais confiáveis para apoiar a tomada de decisões. $\mathrm{O}$ custo de erros associados a essas predições pode, portanto, ser elevado, como, por exemplo, o investimento na conservação de espécies que não estão de fato ameaçadas por mudanças climáticas.

Geralmente, por razões práticas, os pesquisadores selecionam uma técnica ou um algoritmo a partir de um leque variado de alternativas, justificando a sua escolha ao referenciar um ou mais estudos. Porém, vários estudos têm demonstrado que as projeções de modelos podem ser muito variáveis, mesmo considerando um conjunto de modelos com elevada acurácia (Araújo et al., 2005b;
Araújo \& New, 2006; Algar et al., 2009). Enquanto as projeções para o clima atual podem ser testadas com os dados observados, as projeções futuras não podem ser validadas da mesma forma porque não apresentam observações para teste (Hijmans \& Graham, 2006). Assim, métodos para melhorar as incertezas decorrentes destas amplitudes têm sido adotados recentemente e uma das alternativas inclui o uso de vários modelos e técnicas apropriadas para explorar melhor os resultados das diferentes projeções (Araújo et al., 2005b; Araújo \& New, 2006; Diniz-Filho et al., 2009).

As espécies nativas localizadas nas regiões Sul e Sudeste do Brasil e nas áreas de maiores altitudes certamente serão as mais afetadas pela restrição de temperaturas ótimas devido às mudanças climáticas, considerando que nestas localidades os valores de temperatura são mais baixos comparados a outras regiões do Brasil. Os estudos sobre os efeitos das mudanças climáticas no Brasil, de acordo com o último relatório que apresenta diferentes cenários de emissão de gases efeito-estufa (Marengo, 2006), estão apontando para elevações de temperatura em várias regiões e, particularmente, para a região Sul há tendências de incremento nas temperaturas máximas e mínimas anuais e sazonais, além de uma tendência ao aumento de precipitação.

Além disso, os remanescentes de Mata Atlântica nas regiões Sul e Sudeste do Brasil ainda sofrem elevada pressão antrópica, apresentando um elevado número de fragmentos e vários padrões de fragmentação (Morellato \& Haddad, 2000). Para um dado ecossistema, comunidades funcionalmente diversas são mais prováveis de se adaptarem às mudanças climáticas e à variabilidade de clima do que comunidades biologicamente empobrecidas (Brasil, 2007). Dessa forma, as espécies nos fragmentos remanescentes da Mata Atlântica nessas regiões, devem apresentar esse adicional de vulnerabilidade.

As características que aumentam a vulnerabilidade, ou seja, regiões mais frias, com comunidades biologicamente empobrecidas em habitats fragmentados, podem ser encontradas para espécies endêmicas da Mata Atlântica e restritas às regiões Sul e Sudeste, como é o caso da Dicksonia sellowiana (Pres.) Hook., popularmente conhecida como xaxim. Devido à perda de habitats e à exploração comercial sem critérios, a espécie também está na lista oficial das espécies da flora brasileira ameaçadas de extinção (Brasil, 2008a). 
Este estudo teve por objetivo estabelecer e analisar as incertezas de predições sobre o impacto das mudanças climáticas, considerando dois cenários de emissões de gases de efeito estufa futuros, sobre a samambaia arborescente Dicksonia sellowiana. As análises utilizaram modelos de distribuição de espécies e usaram técnicas para mensurar o grau de incerteza nesse tipo de modelagem. Além disso, foram analisados os padrões de distribuição espacial atual e futuro para a espécie em relação aos remanescentes de habitats disponíveis, considerando as áreas de vegetação nativa do bioma Mata Atlântica.

\section{Material e métodos}

\section{Descrição da Espécie}

A espécie Dicksonia sellowiana (Dicksoniaceae) ocorre nos estados de Minas Gerais, São Paulo, Rio de Janeiro, Paraná, Santa Catarina e Rio Grande do Sul, principalmente na Floresta Ombrófila Mista, crescendo em altitudes que variam de $60 \mathrm{~m}$ (ao sul de sua área de distribuição, no RS) até $2.200 \mathrm{~m}$ na Serra de Itatiaia, RJ (Klein, 1963; Fernandes, 1999). Entre as manchas de Floresta Ombrófila Mista, a espécie ocorre também nos os Campos do Planalto, caracterizados como campos limpos, campos sujos e, algumas vezes, em floresta de transição (Klein, 1978).

\section{Conjunto de dados e modelos de distribuição de espécies}

Foram utilizados 70 registros de presença (coordenadas geográficas) gerados por meio de observações de campo e bases de dados na Internet, incluindo MOBOTMissouri Botanical Garden (Tropicos, 2011) e Species Link (Centro de Referência em Informação Ambiental, 2011). Os registros abrangeram toda a área de distribuição geográfica da espécie incluindo os estados do Rio Grande do Sul, Santa Catarina, Paraná, São Paulo e Rio de Janeiro. Apenas para o sul de Minas Gerais não foram obtidos registros.

Para a geração dos modelos foi utilizado o programa Openmodeller (Sutton, 2007) com os algoritmos Bioclim, Climate Space Model, Environmental Distance e Garp (best subsets e single run).

Os modelos incluíram, aleatoriamente, $70 \%$ dos pontos para treino e $30 \%$ para teste. Os pontos de treino foram utilizados para a geração dos modelos enquanto os pontos de teste foram utilizados para os cálculos da estatística da curva ROC (Receiver operating characteristic) e para o índice de Kappa (Fielding \& Bell, 1997).

Como critério de limite de corte para os valores de probabilidade de ocorrência, foi utilizada a média dos valores de probabilidade dos modelos gerados com os dados de clima atual (Liu et al., 2005).

A validação dos modelos para o clima atual foi executada por meio dos seguintes procedimentos: estatística da curva ROC, índice de Kappa, dados de campo e informações de literatura. Os modelos gerados com os dados de clima atual foram comparados e sobrepostos aos dados de distribuição obtidos da literatura (Fernandes, 1999), considerando a área de abrangência da espécie na Floresta Ombrófila Mista e das áreas de transição com outras formações de vegetação (sul de Minas Gerais, São Paulo e Rio de Janeiro). Para os valores da curva ROC foi utilizada a classificação de acurácia descrita por Swets (1988), sendo: excelente para AUC $>0,90$ e bom para $0,80<$ AUC $<0,90$. Para o índice Kappa, valores de $\mathrm{k}>0,75$ foram considerados como excelentes e valores $0,40<\mathrm{k}<0,75$ foram considerados bons (Landis \& Koch, 1977).

Foram utilizados os dados de clima atual e os dados do modelo climático HadCM3 (médias mensais de precipitação e médias das temperaturas máximas e mínimas mensais), além de dados relativos à altitude, todos disponíveis na base de dados Worldclim versão 1.1. Global Climate Surface (Hijmans et al., 2005), na resolução espacial 5 minutos. Esse modelo climático é um dos mais utilizados para avaliação de impactos de mudanças climáticas sobre a biodiversidade (Marengo, 2006). Os dados de altitude na base Worldclim foram gerados por meio de imagens de radar (SRTM- Shuttle Radar Topography Mission). As mesmas variáveis de clima e altitude foram utilizadas nos modelos de distribuição com o clima atual e nos modelos de distribuição com dados climáticos no futuro. A variável altitude foi utilizada considerando que a espécie $D$. sellowiana tem como habitat preferencial as formações de vegetação localizadas no Planalto Meridional (Klein, 1963; Fernandes, 1999). Assim, essa variável também é importante para caracterizar e modelar a distribuição da espécie. Os valores de altitude das áreas de distribuição potencial nos modelos gerados com o clima atual e futuro foram calculados por meio do ambiente do Sistema de Informações Geográficas-SIG (ArcGis 9.2 ESRI, 2004). 
O HadCM3 é um dos modelos utilizados para predições climáticas futuras que consideram diferentes cenários de emissão de gases de efeito estufa (Marengo, 2006). Considerando que o objetivo deste estudo foi avaliar apenas as incertezas associadas aos diferentes modelos de distribuição de espécies, não foram consideradas aqui as análises de incertezas associadas aos diferentes cenários climáticos e aos diferentes modelos de clima.

\section{Cenários climáticos e padrões de uso do solo}

A modelagem incluiu o modelo climático do IPCC HadCM3, em cenários A2 (pessimista) e B2 (otimista), com projeções para o ano 2050. Esses cenários representam uma visão possível do desenvolvimento futuro de emissões de substâncias que têm um efeito radiativo potencial (gases de efeito estufa). Nesses diferentes cenários, as emissões dos gases $\mathrm{CO}_{2}, \mathrm{~N}_{2} \mathrm{O}$, $\mathrm{CH}_{4}$ e $\mathrm{SO}_{2}$ são variáveis e apresentam as seguintes características gerais (Marengo, 2006):

(A2) Cenário pessimista, com um mundo futuro heterogêneo onde a regionalização é dominante, com crescimento populacional alto, embora com menor preocupação em relação ao desenvolvimento econômico rápido e contínuo aumento na concentração de $\mathrm{CO}_{2}$, com valores em torno de $17 \mathrm{GtC}$ (Giga-toneladas de carbono) em 2050;

(B2) Cenário otimista com um mundo futuro onde há ênfase em soluções locais, e também com maior sustentabilidade ambiental, social e econômica. As concentrações de $\mathrm{CO}_{2}$ apresentam ainda tendência de aumento, com valores de emissão em torno de $10 \mathrm{GtC}$ em 2050, porém com emissões menores que o cenário A2. As reduções expressivas de emissão para esse cenário climático são para os gases $\mathrm{SO}_{2}$ e $\mathrm{N}_{2} \mathrm{O}$.

Por meio do ambiente SIG (ArcGis 9.2 ESRI, 2004) foram utilizados os dados oriundos da classificação de remanescentes de vegetação atuais do bioma Mata Atlântica, que inclui as formações florestais, áreas úmidas e áreas antropizadas disponibilizados pelo Ministério do Meio Ambiente/PROBIO (Brasil, 2008b). Esses dados foram sobrepostos aos modelos de distribuição da espécie para a geração dos dados de tamanho dos habitats disponíveis (remanescentes de formações florestais nativas, em $\mathrm{km}^{2}$ ), por meio da intercessão entre os temas.

Medidas de tendência central (medianas) foram calculadas como estratégia de predição de consenso para os modelos gerados (Araújo et al., 2005b). A comparação entre os modelos de distribuição (dados em $\mathrm{km}^{2}$ ) no presente e no futuro para os remanescentes de vegetação (formações florestais) e para as áreas totais (remanescentes de formações florestais e campestres, áreas úmidas e áreas antropizadas) foi feita com análise de variância de Kruskal-Wallis, seguida de teste de Student-Newman-Keuls $(\mathrm{p}<0,05)$.

\section{Resultados e discussão}

Os modelos de distribuição para espécie Dicksonia sellowiana no clima atual apresentaram padrões espaciais semelhantes aos observados em literatura, abrangendo, na maior parte dos modelos, os estados do Paraná, Santa Catarina, norte do Rio Grande do Sul e sul de São Paulo (Figura 1). Considerando a ocorrência da espécie na Floresta Ombrófila Mista (Klein, 1963) e outras formações florestais nos estados de São Paulo, Rio de Janeiro e sul de Minas Gerais (Fernandes, 1999), os melhores resultados incluíram os algoritmos GARP e Bioclim. Porém, apenas para esse último modelo a estatística da curva ROC e o índice de Kappa apresentaram os valores de acurácia mais elevados (Tabela 1). Se considerarmos apenas os valores de referência da área sobre a curva ROC e do índice de Kappa (Swets, 1988; Landis \& Koch, 1977), todos os algoritmos apresentaram eficiência nas projeções para o clima atual. A área sobre a curva ROC (estatística AUC) apresentou valores de acurácia considerados excelentes (AUC $>0,90)$ para quatro algoritmos, e um valor considerado bom $(0,80<\mathrm{AUC}<0,90)$ para o algoritmo GARP-single run (Tabela 1). Os valores do índice de Kappa também apresentaram acurácia excelente $(\mathrm{k}>0,75)$, com exceção do algoritmo Climate Space Model $(0,40<\mathrm{k}<0,75)$. Porém, considerando que a espécie possui área de distribuição bem conhecida, com os melhores modelos derivados do GARP e do Bioclim, as pequenas diferenças observadas nos resultados da estatística da curva ROC e no índice de Kappa não foram suficientes para serem utilizadas como critérios para aferir os melhores modelos. Lobo et al. (2008) observaram problemas associados ao uso da estatística da curva ROC como medida de acurácia, como, por exemplo, a falta de informação sobre a distribuição espacial dos erros. Neste estudo ocorreram maiores valores de acurácia para algoritmos que expandiram 
a área potencial de distribuição da espécie, um pouco mais ao sul, como no caso do modelo gerado pelo algoritmo Environmental Distance (Tabela 1). Essa expansão de área potencial em relação ao algoritmo GARP pode ter influenciado nos menores valores para os erros de omissão, devido aos registros localizados nas áreas marginais mais ao sul da distribuição da espécie, resultando, portanto, em valores de acurácia um pouco mais elevados.
Tabela 1. Valores de acurácia (índice de Kappa e ROC) dos diferentes algoritmos para a distribuição potencial de Dicksonia sellowiana com os dados de clima atual.

\begin{tabular}{lcc}
\hline \multicolumn{1}{c}{ Algoritmo } & AUC-ROC & Kappa \\
\hline Bioclim & 1,00 & 1,00 \\
Climate Space Model & 0,92 & 0,74 \\
EnvironmentalDistance - Chebychev & 1,00 & 0,92 \\
GARP-single run & 0,88 & 0,84 \\
GARP-best subsets & 0,90 & 0,84 \\
\hline AUC-ROC - estatística da área sob a curva ROC; Kappa: índice Kappa.
\end{tabular}

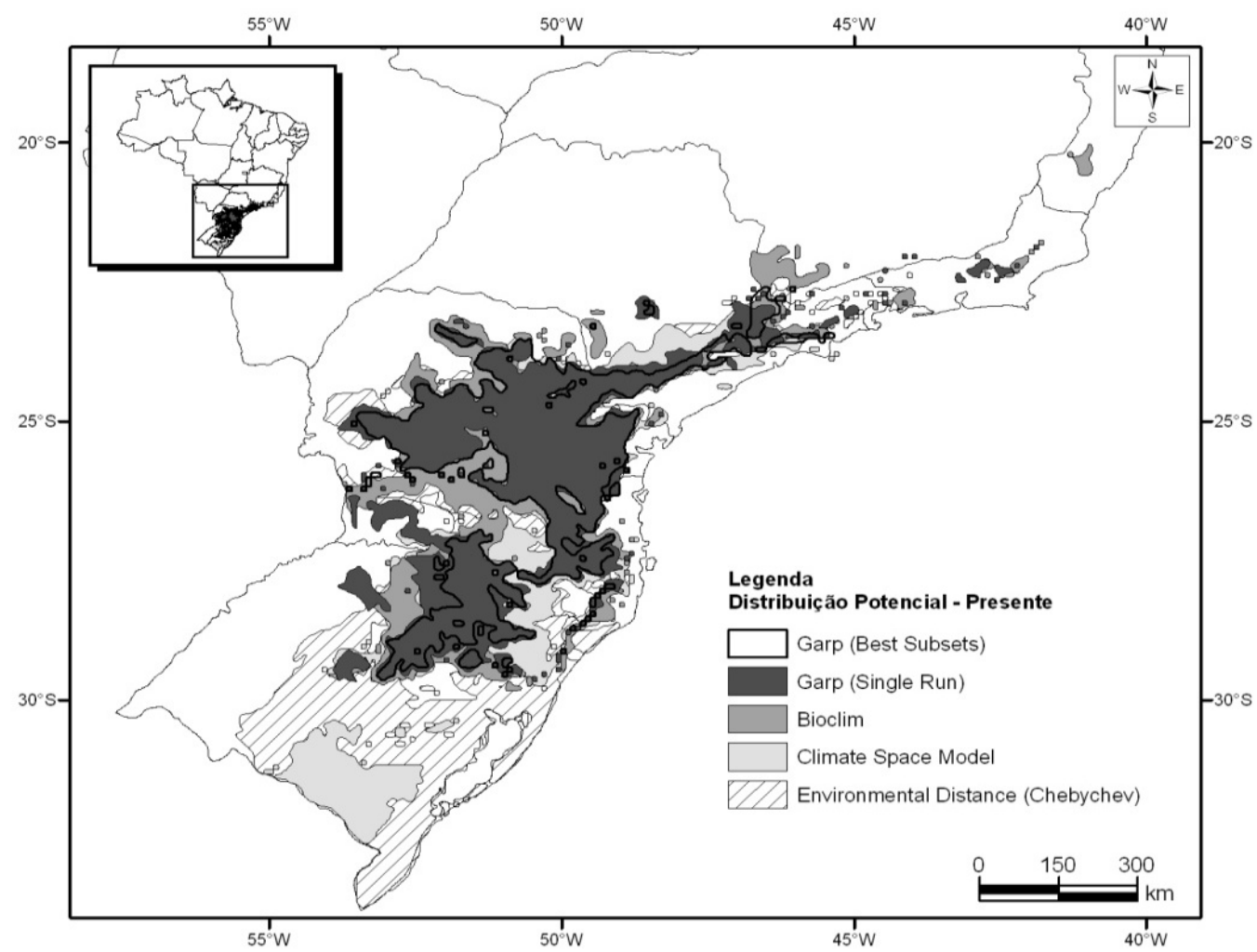

Figura 1. Área de distribuição potencial de Dicksonia sellowiana no clima atual, considerando diferentes algoritmos (Environmental Distance, Climate Space Model, Bioclim e GARP).

As áreas de distribuição potencial para o clima atual apresentaram valor de mediana igual a $222.397 \mathrm{~km}^{2}$ (primeiro quartil= 184.619; terceiro quartil=242.582), enquanto a área de distribuição potencial no cenário de clima futuro A2 apresentou retração, com valor de mediana igual a $62.684 \mathrm{~km}^{2}$ (primeiro quartil=44.914; terceiro quartil=82.723). Para o cenário de clima futuro B2 o valor de mediana foi igual a $74.984 \mathrm{~km}^{2}$ (primeiro quartil $=57.608$; terceiro quartil $=119.821$ ) (Figura 2). Mesmo considerando os valores bons e excelentes de acurácia com os dados de clima atual
(Tabela 1), os elevados valores de amplitude gerados com as projeções para o clima no futuro corroboraram a necessidade do uso de técnicas de consenso para a redução das incertezas associadas a este tipo de modelagem (Araújo et al., 2005b; Araújo \& New, 2006; Algar et al., 2009). O uso de um único algoritmo para projeções de distribuição no futuro poderia induzir a erros consideráveis, e medidas de tendência central com vários modelos podem quantificar melhor essa incerteza e indicar cenários mais confiáveis sobre os impactos de mudanças climáticas e sobre a vulnerabilidade desta 
espécie. Apesar da retração, os valores das áreas de distribuição potencial de $D$. sellowiana para o ano 2050 não apresentaram diferença significativa em relação às áreas de distribuição com o clima atual $(H=5,54 ; p>0,05)$. Entre os dois cenários climáticos futuros, as concentrações crescentes dos gases $\mathrm{CO}_{2}$ e $\mathrm{CH}_{4}$ para o cenário $\mathrm{B} 2$, embora menores que para o cenário A2 (Marengo, 2006), devem manter os valores de temperatura em níveis que afetarão a distribuição de forma similar nos dois cenários climáticos.

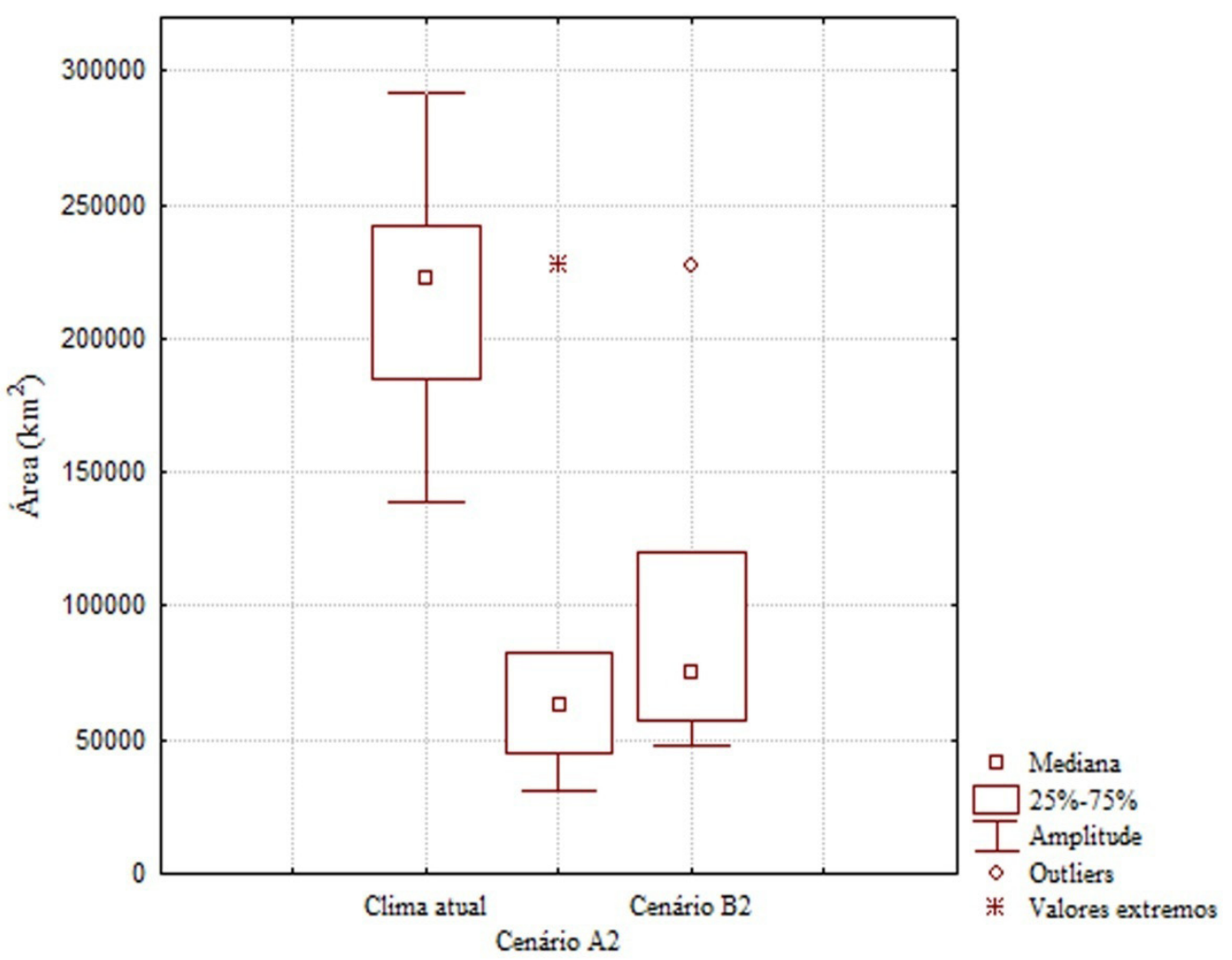

Figura 2. Box-plot representando a área de distribuição potencial de Dicksonia sellowiana considerando o clima atual e os cenários climáticos A2 e B2 do modelo HadCM3 para o ano 2050.

Porém, considerando apenas as classes de uso do solo caracterizadas como áreas mais efetivas para a manutenção de populações da espécie, como os remanescentes de Floresta Estacional, Floresta Ombrófila, formações pioneiras e vegetação secundária, o valor de mediana é igual a $67.987 \mathrm{~km}^{2}$ (primeiro quartil=54.318; terceiro quartil=75.908) para os dados de clima atual, enquanto a área de distribuição potencial no cenário climático $\mathrm{A} 2$ apresentou retração com valor de mediana igual a $21.894 \mathrm{~km}^{2}$ (primeiro quartil $=14.856$; terceiro quartil $=28.168$ ), e para o cenário de clima futuro B2 o valor de mediana foi igual a $27.689 \mathrm{~km}^{2}$ (primeiro quartil $=21.998$; terceiro quartil=41. 866) (Figura 3). Os resultados apresentaram diferenças significativas entre as projeções de área com o clima atual e em comparação com as áreas em cenários climáticos futuros $(\mathrm{H}=6,18$; $\mathrm{p}<0,05)$. A redução de áreas potenciais superior a $50 \%$ em ambos os cenários corrobora a vulnerabilidade da espécie ao aquecimento global, restrita a poucas manchas de Mata Atlântica disponíveis. Não houve diferenças significativas para as áreas comparando-se os cenários $\mathrm{A} 2$ e B2 $(\mathrm{H}=5,54 ; \mathrm{p}>0,05)$. Portanto, mesmo o cenário climático mais otimista deve representar uma retração expressiva de habitats disponíveis para $D$. sellowiana. Colombo \& Joly (2010) verificaram reduções de $25 \%$ (cenário otimista) a 50\% (cenário pessimista) para as áreas potenciais de 38 espécies de Mata Atlântica utilizando o algoritmo GARP, com deslocamento de habitats para áreas mais frias ao sul desse bioma. Com o mesmo algoritmo, Siqueira \& Peterson (2003) 
verificaram reduções superiores a $50 \%$ na área de distribuição potencial para 162 espécies arbóreas do Cerrado, considerando cenários de aumento de $0,5 \%$ a $1 \%$ de $\mathrm{CO}_{2}$ por ano na atmosfera nos próximos 50 anos.

Ambientes com elevada fragmentação, como neste estudo, tornam as projeções futuras de disponibilidade de habitats mais incertas (Guisan \& Thuiller, 2005), considerando as barreiras derivadas do antropismo que podem dificultar a dispersão e colonização de novas áreas. Assim, a efetiva manutenção de populações viáveis de $D$. sellowiana nos habitats remanescentes pode ser ainda mais restritiva.

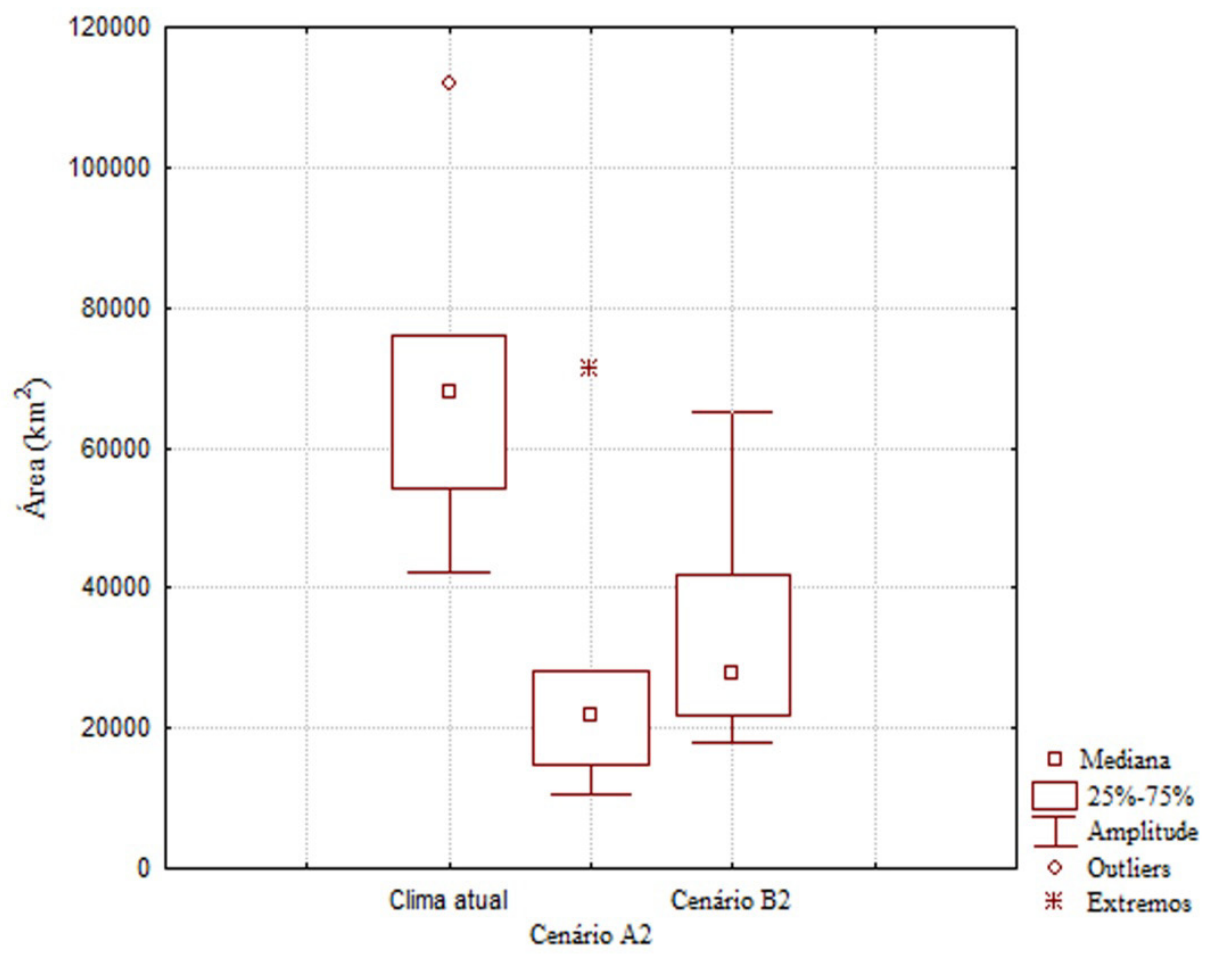

Figura 3. Box-plot representando a área de distribuição potencial dos remanescentes de Floresta Estacional, Floresta Ombrófila, formações pioneiras e vegetação secundária para Dicksonia sellowiana considerando o clima atual e os cenários climáticos A2 e B2 do modelo HadCM3 para o ano 2050.

A média da altitude obtida do modelo para o presente foi de $695,4 \pm 84,1$ m. Já a média da altitude dos modelos futuros variou de $887,8 \pm 165,5$ m (cenário A2) a $844,2 \pm$ 164,2 $\mathrm{m}$ (cenário B2). Muitas espécies têm respondido às mudanças climáticas no século passado com migrações para cotas de altitude mais elevadas (Parmesan \& Yohe, 2003). No caso de D. sellowiana a restrição de populações nas áreas mais elevadas parece ser um cenário provável para essa espécie (Figura 4). Apenas os modelos de distribuição com o cenário climático B2 são representados na Figura 4, considerando o padrão de distribuição muito similar ao cenário A2.

Alguns estudos têm questionado o uso de modelagens com envelopes bioclimáticos para a distribuição espacial de espécies e a sua dinâmica no tempo, considerando que outros fatores, como as interações bióticas entre espécies, como competição, predação, simbiose (Davis et al., 1998; Guisan \& Thuiller, 2005; Araújo \& Luoto, 2007) e adaptações evolutivas (Pearson \& Dawson, 2003; Harte et al., 2004; Diniz-Filho \& Bini, 2008) podem ser também muito importantes para explicar essa dinâmica. Interações bióticas de fato podem alterar os padrões de distribuição de $D$. sellowiana no futuro, mas não podem ser avaliadas pelos modelos utilizados neste estudo. As adaptações evolutivas rápidas ou a redução populacional, acompanhadas de retrações de área para cotas de altitude mais elevadas, parecem ser as possibilidades mais prováveis para a espécie 
D. sellowiana, com o agravante de que as adaptações evolutivas rápidas podem ser bastante limitadas (Wiens \& Graham, 2005). Embora a redução de habitats disponíveis seja um fator chave para o declínio de espécies, alguns casos de persistência em longo prazo em habitats reduzidos indicam a necessidade de investigação da escala de sensibilidade para cada espécie, ou a capacidade de manutenção de populações viáveis em habitats pequenos e fragmentados (Thuiller et al., 2004).

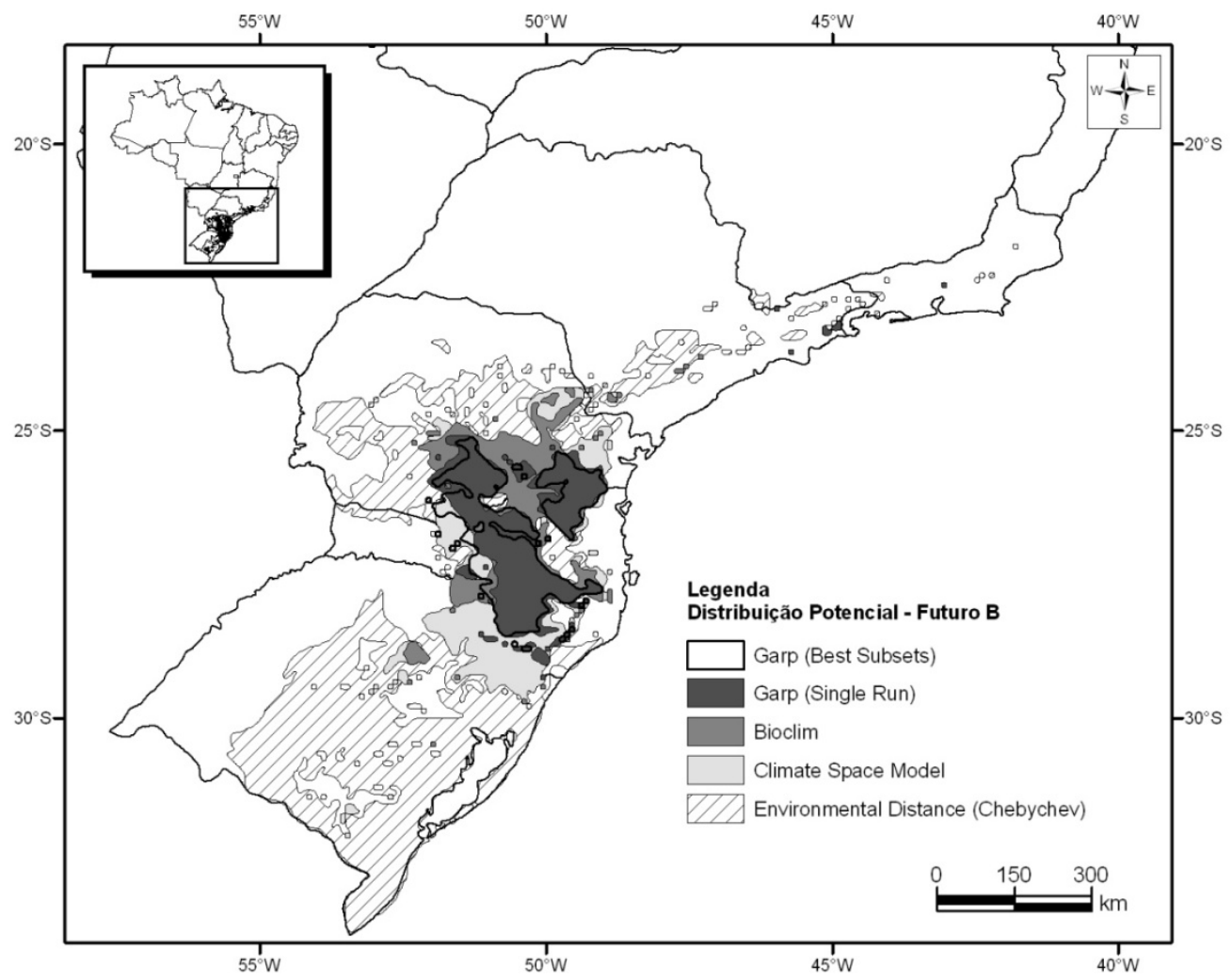

Figura 4. Área de distribuição potencial de Dicksonia sellowiana no cenário climático B2 (modelo HadCM3), considerando diferentes algoritmos (Environmental Distance, Climate Space Model, Bioclim e GARP).

\section{Conclusões}

Os resultados obtidos para a distribuição espacial de D. sellowiana em cenários climáticos futuros reforçaram a necessidade de uso de predições com técnicas que busquem um consenso entre as várias opções disponíveis. A amplitude das predições das áreas de distribuição potencial observadas para essa espécie corroborou as incertezas associadas aos vários algoritmos e a validade do uso de técnicas exploratórias para reduzir o erro dessas variações, como as medidas de tendência central.
Com o uso de abordagens para reduzir as incertezas associadas aos diferentes algoritmos, as técnicas de modelagem de distribuição espacial podem constituir ferramentas importantes no processo de entendimento de padrões futuros de clima sobre a vulnerabilidade ao aquecimento global de espécies de clima mais frios e em habitats antropizados, como no caso de D. sellowiana. Os modelos gerados neste estudo corroboraram a vulnerabilidade dessa espécie em relação ao aquecimento global, e mesmo o cenário climático mais otimista deve representar uma retração expressiva de áreas para $D$. sellowiana. 


\section{Agradecimentos}

Os autores agradecem à Marinez Ferreira de Siqueira, pelas sugestões sobre métodos; à Funarbe, pela bolsa de iniciação científica concedida à Mahalia Sojo Cardoso, e ao CNPq-PIBIC, pela concessão de bolsa de iniciação científica ao Rafael Walter de Albuquerque.

\section{Referências}

ALGAR, A. C.; KHAROUBA, H. M.; YOUNG, E. R.; KERR, J. T. Predicting the future of species diversity: macroecological theory, climate change, and direct tests of alternative forecasting methods. Ecography, Copenhagen, n. 32, p.22-33, 2009.

ARAÚJO, M. B.; PEARSON, R. G.; THUILLER, W.; ERHARD, M. Validation of species-climate impacts models under climate change. Global Change Biology, Malden, n. 11, p. 1504-1513, 2005a.

ARAÚJO, M. B.; WHITTAKER, R. J.; LADLE, R. J.; ERHARD, M. Reducing uncertainty in projections of extinction risk from climate change. Global Ecology and Biogeography, Malden, n. 14, p. 529-538, 2005 b.

ARAÚJO, M. B.; THUILlER, W.; PEARSON, R. G. Climate warming and decline of amphibians and reptiles in Europe. Journal of Biogeography, Hoboken, n. 33, p. 1712-1728, 2006.

ARAÚJO, M. B.; NEW, M. Ensemble forecasting of species distributions. Trends in Ecology and Evolution, Oxford, n. 22, p. 42-47, 2006.

ARAÚJO, M. B.; LUOTO, M. The importance of biotic interactions for modelling species distributions under climate change. Global Ecology and Biogeography, Malden, n. 16, p. 743-753, 2007.

BEAUMONT, L. J.; PITMAN, A. J.; POULSEN, M.; HUGHES, L. Where will species go? Incorporating new advances in climate modeling into projections of species distributions. Global Change Biology, Malden, n. 13, p. 1368-1385, 2007.

BRASIL. Ministério do Meio Ambiente. Inter-relações entre mudanças climáticas e biodiversidade. Brasília, DF: Ministério do Meio Ambiente, 2007. (Biodiversidade, 28).

BRASIL. Ministério do Meio Ambiente. Instrução Normativa $n^{\circ}$ 6 de 23 de setembro de 2008. Lista as espécies da flora brasileira ameaçadas de extinção e com deficiência de dados. Diário Oficial [da] República Federativa do Brasil, Brasília, DF, n. 185, seção 1, p. 75-83, 2008a.

BRASIL. Ministério do Meio Ambiente. Mapas de cobertura vegetal dos biomas brasileiros. Brasília, DF: Ministério do Meio Ambiente, 2008b.

COLOMBO, A. F.; JOLY, C. A. Brazilian Atlantic Forest lato sensu: the most ancient Brazilian forest, and a biodiversity hotspot, is highly threatened by climate change. Brazilian Journal of Biology, São Carlos, v. 70, n. 3, p. 697-708.

CENTRO DE REFERÊNCIA EM INFORMAÇÃO AMBIENTAL. Disponível em: $<$ http//www.cria.org.br $>$. Acesso em: 10 nov. 2008.
DAVIS, A.J.; JENKINSON, L.S.; LAWTON, J.L.; SHORROCKS, B.; WOOD, S. Making mistakes when predicting shifts in species range in response to global warming. Nature, New York, n. 391, p. 783-786, 1998.

DINIZ-FILHO, J. A. F.; BINI, L. M. Macroecology, global change and the shadow of forgotten ancestors. Global Ecology and Biogeography, Malden, n. 17, p. 11-17, 2008.

DINIZ-FILHO, J. A. F.; BINI, L. M.; RANGEL, T. F.; LOYOLA, R. D.; HOF, C.; NOGUÉS-BRAVO, D.; ARAÚJO, M. B. Partitioning and mapping uncertainties in ensembles of forecasts of species turnover under climate change. Ecography, Hoboken, n. 32, p. 897-906, 2009.

\section{ESRI. 2004. ArcGis 9.2. New York.}

FERNANDES, I. Taxonomia dos representantes de Dicksoniaceae no Brasil. Revista de Pesquisas, São Paulo, v. 50, 1999.

FIELDING, A. H.; BELL, J. F. A review of methods for the assessment of prediction errors in conservation presence/absence models. Environmental Conservation, Cambridge, n. 24, p. 3849, 1997.

GUISAN, A.; THUILLER, W. Predicting species distribution: offering more than simple habitat models. Ecology Letters, Hoboken, n. 8, p. 993-1009, 2005.

HARTE, J.; OSTlinG, A.; GREEN, J. L.; KINZIG, A. Climate change and extinction risk. Nature, New York, n. 430, p. 34, 2004.

HIJIMANS, R. J.; CAMERON, S. E.; PARRA, J. L.; JONES, P. G.; JARVIS, A. Very high resolution interpolated climate surfaces for global land areas. International Journal of Climatology, Hoboken, n. 25, p. 1965-1978, 2005.

HIJIMANS, R. J.; GRAHAM, C. H. The ability of climate envelope models to predict the effect of climate change on species distribution. Global Change Biology, Hoboken, n. 12, p. 2272-2281, 2006.

HUTCHINSON, G. E. Concluding remarks. Cold Spring Harbor Symposia on Quantitative Biology, Cold Spring, n. 22, p. 415427, 1957.

KLEIN, R. M. Observações e considerações sobre a vegetação do Planalto Nordeste Catarinense. Sellowia, Itajaí, n. 15, Separata, 1963.

KLEIN, R. M. Mapa fitogeográfico do estado de Santa Catarina. Itajaí: Herbário Barbosa Rodrigues, 1978. (Flora Ilustrada Catarinense).

LANDIS, J. R.; KOCH, G. C. The measurement of observer agreement for categorical data. Biometrics, Arlington, n. 33, p. 159-174, 1977.

LIU, C.; BERRY, P. M.; DAWSON, T. P.; PEARSON, R. G. Selecting thresholds of occurrence in the prediction of species distributions. Ecography, n. 28, p. 385-393, 2005.

LOBO, J. M.; VALVERDE-JIMENEZ, A.; REAL, R. AUC: a misleading measure of the performance of predictive distribution models. Global Ecology and Biogeography, Malden, n. 17, p. 145-151, 2008.

MARENGO, J.A. Mudanças climáticas globais e seus efeitos sobre a biodiversidade. Brasília, DF: Ministério do Meio Ambiente, 2006. (Biodiversidade, 26). 
MORELlATO, P. C.; HADDAD, C. F. B. The Brazilian Atlantic Forest. Biotropica, Hoboken, n. 32, p. 786-792, 2000.

PARMESAN, C.; YOHE, G. A globally coherent fingerprint of global climate impacts across natural systems. Nature, New York, n. 421, p. 37-42, 2003.

PEARSON, R. G.; DAWSON, T. P. Predicting the impacts of climate change on the distribution of species: are the bioclimate envelope models useful? Global Ecology and Biogeography, n. 12, p. 361371, 2003.

PEARSON, R. G.; THUILLER, W.; ARAÚJO, M. B.; MARTINEZMEYER, E.; BROTONS, L.; MACCLEAN, C.; MILES, L.; SEGURADO, P.; DAWSON, T. P.; LEES, D. C. Model-based uncertainty in species range prediction. Journal of Biogeography, Hoboken, n. 33, p. 1704-1711, 2006.

PETERSON, A. T.; ORTEGA-HUERTA, M. A.; BARTLEY, J.; SANCHÉZ-CORDEIRO, V.; SOBERÓN, J.; BUDDEMEIER, R. H.; STOCKWELL, D. R. B. Future projections for Mexican faunas under global climate change scenarios. Nature, New York, n. 416, p. 626-629, 2002.

THOMAS, C. D.; CAMERON, A.; GREEN, R. E.; BAKKENES, M.; BEAUMONT, L. J.; COLLINGHAM, Y. C.; ERASMUS, B. F. N.; SIQUEIRA, M. F.; GRAINGER, A.; HANNAH, L.; HUGHES, L; HUNTLEY, B.; JAARSVELD, A. S.; MIDGLEY, G. F.; MILES, L.; ORTEGA-HUERTA, M. A.; PETERSON, A. T.; PHILLIPS, O. L.; WILLIAMS, S. E. Extinction risk from climate change. Nature, New York, n. 427, p. 145-148, 2004.
TROPICOS. Missouri Botanical Garden. Disponível em: <http// www.tropicos.org >. Acesso em: 05 nov. 2008.

SIQUEIRA, M. F.; PETERSON, A. T. Consequences of global climate change for geographic distributions of Cerrado tree species. Biota Neotropica, São Paulo, v. 3, n. 2, p. 1-14, 2003.

SUTTON, T. Openmodeller. Disponível em: $<$ http://openmodeller. sourceforge.net/>. Acesso em: 6 jul. 2008.

SWETS, K. A. Measuring the accuracy of diagnostic systems. Science, Washington, DC, n. 240, p. 1285-1293, 1988.

THUILLER, W. Patterns and uncertainties of species range shifts under climate change. Global Change Biology, Hoboken, n. 10, p. 2020-2027, 2004.

WIENS, J. J.; GRAHAM, C. H. Niche conservatism: integrating evolution, ecology and conservation biology. Annual Review of Ecology and Evolution Systematics, Palo Alto, n. 36, p. 519-539, 2005. 